\title{
Study on mechanism of the Hanshi Zufei syndrome formula for COVID-19 based on network pharmacology
}

\section{Wenjiang Zheng}

Guangzhou University of Chinese Medicine

\section{Yongshi Ni}

Guangzhou University of Chinese Medicine

\section{Qian Yan}

Guangzhou University of Chinese Medicine

\section{Ying Huang}

Guangzhou University of Chinese Medicine

Jiqiang Li

Guangzhou University of Chinese Medicine

Xiaohong Liu

Guangzhou University of Chinese Medicine

Yong Jiang ( $\sim$ jiangyongszzxy@163.com )

Shenzhen People's Hospital https://orcid.org/0000-0002-3609-2280

\section{Research}

Keywords: COVID-19, network pharmacology, Hanshi Zufei syndrome formula, flavonoids, targets, signaling pathways

Posted Date: July 21 st, 2020

DOI: https://doi.org/10.21203/rs.3.rs-41445/v1

License: (a) (1) This work is licensed under a Creative Commons Attribution 4.0 International License. Read Full License 


\section{Abstract \\ Objective}

To study the mechanisms of Hanshi Zufei syndrome formula (HSZF) for coronavirus disease 2019 (COVID-19) using network pharmacology.

\section{Methods}

We screened the potential active constituents (oral bioavailability $[\mathrm{OB}] \geq 30 \%$, drug-likeness $[\mathrm{DL}] \geq 0.18$ ) of HSZF using the Traditional Chinese Medicine Systems Pharmacology (TCMSP) database, predicted targets using the Swiss TargetPrediction database, constructed a pharmacologically active-compoundpotential-target network and a protein-protein interaction (PPI) network using Cytoscape software, and used the org.Hs.eg.db and ClusterProfiler data packages in $\mathrm{R}$ language software to perform gene ontology (GO) biological-function analysis and Kyoto Encyclopedia of Genes and Genomes (KEGG) pathway enrichment analysis on the potential targets of HSZF.

\section{Results}

A total of 170 effective active constituents, such as Genkwanin, diosmetin, wogonin, quercetin, kaempferol, luteolin, and herbacetin were obtained through oral OB and DL screenings. These constituents act on targets such as kininogen-1 (KNG1), epidermal growth factor receptor (EGFR), Caspase-3 (CASP3), signal transducer and activator of transcription 3 (STAT3), EStrogen Receptor 1 (ESR1), angiotensin-converting enzyme 2 (ACE2), and myeloperoxidase (MPO); participate in biological processes such as cellular metal ion homeostasis, calcium ion homeostasis, unsaturated fatty acid metabolism, and positive regulation of phospholipase activity; and treat COVID-19 through pathways such as apoptosis, calcium signaling, phospholipase D signaling, arachidonic acid metabolism, platelet activation, renin-angiotensin system (RAS), and inflammatory-mediator regulation of transient receptor potential (TRP) channels.

\section{Conclusion}

Flavonoids are important in the mechanism by which HSZF treats COVID-19. Inhibition of inflammatory response and regulation of both immune function and cell apoptosis might be important mechanisms of HSZF for this disease.

\section{Introduction}

At the end of December 2019, coronavirus disease 2019 (COVID-19), caused by severe acute respiratory syndrome coronavirus 2 (SARS-CoV-2), broke out in Wuhan, Hubei Province, China. It then spread rapidly 
throughout China and other countries in the world. COVID-19 has become an urgent public-health event of international concern, with a total of more than 100,000 confirmed cases worldwide. The mild form of COVID-19 manifests only as low fever and mild fatigue without pneumonia, while severe cases can quickly progress to acute respiratory distress syndrome (ARDS), metabolic acidosis that is difficult to correct, multiple-organ failure (MOF), and even death. At present, no specific antiviral drug has been shown to be effective against COVID-19[1-3].

TCM has accumulated a wealth of experience in the fight against epidemics for thousands of years and has achieved good clinical results in the prevention and treatment of new infectious diseases[4]. Therefore, TCM treatments are included in the Diagnosis and Treatment Plan for COVID-19 (trial versions $4,5,6$, and 7) jointly issued by the National Health Commission of the People's Republic of China and the National Administration of Traditional Chinese Medicine. In the Diagnosis and Treatment Plan for COVID19 (trial version 7), Hanshi Zufei syndrome formula (HSZF) is widely used in the treatment of moderate COVID-19 and mainly aimed at patients with symptoms such as low fever, dry cough with little phlegm, fatigue, chest tightness, and diarrhea[5]. The formula consists of 9 Chinese herbs: $15 \mathrm{~g}$ rhizome atractylodes, $10 \mathrm{~g}$ tangerine peel, $10 \mathrm{~g}$ bark of magnolia, $10 \mathrm{~g}$ wrinkled giant hyssop herb, $6 \mathrm{~g}$ tsaoko amomum fruit, $6 \mathrm{~g}$ raw herba ephedrae, $10 \mathrm{~g}$ incised notopterygium, $10 \mathrm{~g}$ ginger, and $10 \mathrm{~g}$ betel nut. The components are mutually compatible, dispelling cold pathogens outside and stagnation of dampness inside the body. In view of the outstanding clinical efficacy of this formula, research on its anti-COVID-19 mechanism can provide basic support for deeper understanding of TCM in the prevention and treatment of COVID-19.

At the molecular level, TCM compounds play a comprehensive and holistic role in regulating the body via multiple components and targets. Network pharmacology is an effective method of studying the mechanisms of such drugs[6, 7]. In our study, we used a network pharmacology method to explore the active constituents and molecular mechanisms of HSZF in COVID-19 treatment. We made a list of the compounds contained in HSZF and their corresponding targets through database searches, constructed and analyzed the target network, determined the important targets and submodules in the network, performed functional-enrichment analysis on the important targets and submodules, and revealed the possible action mode of HSZF in the treatment of COVID-19.

\section{Materials And Methods}

\subsection{Collection of chemical constituents in HSZF}

From the Traditional Chinese Medicine Systems Pharmacology (TCMSP) database (http://lsp.nwu.edu.cn/tcmsp.php)[8], we retrieved chemical constituents of 9 Chinese herbs that are components of HSZF: rhizome atractylodes, tangerine peel, bark of magnolia, wrinkled giant hyssop herb, tsaoko amomum fruit, herba ephedrae, incised notopterygium, ginger, and betel nuts. We obtained the molecular structures of their chemical compositions from the PubChem database 
(http://pubchem.ncbi.nlm.nih.gov/) and saved them in canonical simplified molecular-input line-entry system (SMILES) format.

\subsection{Active-constituent screening}

Absorption, Distribution, Metabolism, and Excretion (ADME) evaluation of drugs is a key part of drug screening and research and development. ADME is the process by which the body absorbs, distributes, metabolizes, and excretes exogenous compounds. We used ADME to evaluate the effects and TCM constituents on the human body and their risks [9]. Oral bioavailability (OB) and drug-likeness (DL) are the most common pharmacokinetic parameters for drug screening[10, 11]; we used $O B \geq 30 \%$ and $D L \geq$ 0.18 as the screening conditions for active constituents of HSZF.

\subsection{Predicting the targets of HSZF}

The SwissTargetPrediction database (http://www.swisstargetprediction.ch/)[12]can accurately predict targets of active constituents based on similarity between the two- and three-dimensional structures of the molecule and the known ligand. We imported the abovementioned SMILES format file into the SwissTargetPrediction database. Using humans as the research species, we obtained the targets of the compounds and saved them in. csv format. Potential targets of HSZF were obtained after integration and deduplication.

\subsection{Acquisition of targets of COVID-19}

There is no clear therapeutic target for COVID-19 at present. It is reported that the mechanism by which SARS-CoV-2 infects type II alveoli is the interaction of spike (S) protein with human renin and angiotensinconverting enzyme 2 (ACE2), thereby infecting type II alveolar cells and resulting in the occurrence of pneumonia. Therefore, based on single-cell sequencing results of colon epithelial cells in a previous study[13], we extracted genes co-expressed with ACE2 and performed standardized conversion of gene names in the original files to match human-related targets. By mapping these genes to the abovementioned potential targets of HSZF, we obtained the potential targets of HSZF in the treatment of COVID-19.

\subsection{Building a network of active compounds and potential targets}

We introduced the obtained active compounds and potential targets into Cytoscape software version 3.7.0 (National Resource for Network Biology)[14] and drew a network of active compounds and potential targets.

\subsection{Building a protein-protein interaction (PPI) network}

We introduced potential targets of HSZF in the treatment of COVID-19 into the Search Tool for the Retrieval of Interacting Genes/Proteins (STRING; http://stringdb.org/) [15]. For species, we selected humans. We analyzed PPI between targets, imported the results into Cytoscape via the Cytohubba[16] plug-in for network topology analysis, and used Cytoscape for visual processing. 


\subsection{Gene function and pathway enrichment analysis of targets}

We used the org.Hs.eg.db and ClusterProfiler data packages of R language software version 3.5.2 (Ihaka and Gentleman, 1996)[17] to perform gene ontology (GO) enrichment analysis of gene function [18] and Kyoto Encyclopedia of Genes and Genomes (KEGG) signaling pathways[19] of the common targets obtained. We retained results for which $P \leq 0.05$ and obtained GO and KEGG signaling pathway histograms. Finally, we used Cytoscape to construct a target-signal pathway network diagram to further illustrate the roles of targets and signaling pathways in HSZF in the treatment of COVID-19.

\section{Results}

\subsection{Potential active constituents in HSZF}

We used the TCMSP database to search for compounds of 9 Chinese herbs in HSZF. There were 49 compounds in rhizome atractylodes, 63 in tangerine peel, 139 in bark of magnolia, 94 in wrinkled giant hyssop herb, 59 in tsaoko amomum fruit, 363 in herba ephedrae, 185 in incised notopterygium, 265 in ginger, and 52 in betel nut. Using $\mathrm{OB} \geq 30 \%$ and $\mathrm{DL} \geq 0.18$ for further screening, we obtained, without removing duplicates, a total of 86 potential active constituents: 9 in rhizome atractylodes, 5 in tangerine peels, 2 in bark of magnolia, 11 in wrinkled giant hyssop herb, 8 in tsaoko amomum fruit, 23 in herba ephedrae, 15 in incised notopterygium, 5 in ginger, and 8 in betel nuts.

\subsection{Prediction of potential targets}

We used the SwissTargetPrediction database to predict potential targets of the 70 active constituents obtained after removing duplicates. Of these 70 compounds, 50 had detectable targets, yielding a total of 709 potential targets. Single-cell sequencing of colon epithelial cells identified 5556 genes co-expressed with ACE2. After standardized conversion of the gene names in the original files, we matched a total of 3558 human targets. Next, we plotted 709 component targets and 3558 COVID-19 targets in a Wayne diagram. As shown in Fig. 1, we obtained 170 overlapped targets, which were the relevant targets of the active TCM constituents that might act on COVID-19.

\subsection{Construction and analysis of pharmacologically active- constituent-key target network}

We imported the active constituents and potential targets into Cytoscape and its Network Analyzer tool for network construction and obtained an active-compound-potential-target network (Fig. 2).

Pharmaceutically active constituents are shown in green, targets in red. As can be seen in Table 1 and Fig. 2, 10 pharmacologically active constituents had a degree value $>30$ : pectolinarigenin, genkwanin, 5hydroxy-7,4'-dimethoxyflavanon, diosmetin, wogonin, quercetin, kaempferol, pachypodol, luteolin, and 
herbacetin. These constituents fully reflected the characteristics of multi-component and multi-target TCM. 
Table 1

The 47 main active constituents of Hanshi Zufei syndrome formula (obtained using Network Analyzer in Cytoscape).

\begin{tabular}{|c|c|c|c|c|c|}
\hline No. & Name & Degree & No. & Name & Degree \\
\hline 1 & Pectolinarigenin & 36 & 25 & $\begin{array}{l}\text { 2-Hydroxyisoxypropyl-3-hydroxy- } \\
\text { 7-isopentene-2,3- } \\
\text { dihydrobenzofuran-5-carboxylic }\end{array}$ & 19 \\
\hline 2 & Genkwanin & 34 & 26 & Bergaptin & 19 \\
\hline 3 & $\begin{array}{l}\text { 5-Hydroxy-7,4'- } \\
\text { dimethoxyflavanon }\end{array}$ & 34 & 27 & Mandenol & 18 \\
\hline 4 & Diosmetin & 34 & 28 & isoimperatorin & 16 \\
\hline 5 & wogonin & 34 & 29 & Phellopterin & 15 \\
\hline 6 & quercetin & 33 & 30 & NSC63551 & 13 \\
\hline 7 & kaempferol & 33 & 31 & sitosterol & 11 \\
\hline 8 & pachypodol & 32 & 32 & Eucalyptol & 11 \\
\hline 9 & luteolin & 32 & 33 & beta-sitosterol & 11 \\
\hline 10 & Herbacetin & 31 & 34 & 24-Ethylcholest-4-en-3-one & 11 \\
\hline 11 & $\begin{array}{l}\text { 5,7-dihydroxy-2-(3-hydroxy-4- } \\
\text { methoxyphenyl)chroman-4- } \\
\text { one }\end{array}$ & 30 & 35 & poriferast-5-en-3beta-ol & 11 \\
\hline 12 & Cnidilin & 28 & 36 & phenanthrone & 10 \\
\hline 13 & 6-methylgingediacetate2 & 27 & 37 & Neohesperidin & 9 \\
\hline 14 & naringenin & 26 & 38 & Stigmasterol & 9 \\
\hline 15 & Truflex OBP & 26 & 39 & Procyanidin B1 & 9 \\
\hline 16 & ZINC03860434 & 26 & 40 & beta-sitosterol 3-0-glucoside_qt & 6 \\
\hline 17 & WLN: 60VR BVO6 & 26 & 41 & $\begin{array}{l}\text { (4E,6E)-1,7-bis(4- } \\
\text { hydroxyphenyl)hepta-4,6-dien-3- } \\
\text { one }\end{array}$ & 5 \\
\hline 18 & eriodictyol & 24 & 42 & nobiletin & 4 \\
\hline 19 & EPA & 23 & 43 & campest-5-en-3beta-ol & 4 \\
\hline 20 & $\begin{array}{l}\text { 8-geranoxy-5- } \\
\text { methoxypsoralen }\end{array}$ & 22 & 44 & 6'-Feruloylnodakenin & 4 \\
\hline 21 & Dihydrocapsaicin & 21 & 45 & Citromitin & 3 \\
\hline 22 & DNOP & 21 & 46 & Demethylfuropinnarin & 2 \\
\hline
\end{tabular}




\begin{tabular}{|llllll|}
\hline No. & Name & Degree & No. & Name & Degree \\
\hline 23 & Ammidin & 20 & 47 & Acanthoside B & 1 \\
\hline 24 & irisolidone & 19 & & & \\
\hline
\end{tabular}

\subsection{Network topology analysis}

Our network topology analysis showed network parameters of a total 164 targets. The network relationships of the targets are displayed visually in Table 2 and Fig. 3. 
Table 2

Network topology analysis results (via the Cytohubba plug-in for Cytoscape).

\begin{tabular}{|c|c|c|c|c|c|c|c|c|}
\hline No. & Target & Degree & No. & Target & Degree & No. & Target & Degree \\
\hline 1 & KNG1 & 47 & 56 & QRFPR & 11 & 111 & KCNJ1 & 5 \\
\hline 2 & EGFR & 46 & 57 & HCRTR2 & 11 & 112 & SCN4A & 5 \\
\hline 3 & CASP3 & 41 & 58 & NTRK2 & 11 & 113 & CMA1 & 5 \\
\hline 4 & STAT3 & 34 & 59 & $\mathrm{NR} 1 \mathrm{H} 4$ & 11 & 114 & PIM2 & 5 \\
\hline 5 & ESR1 & 29 & 60 & BTK & 11 & 115 & SLC2A2 & 5 \\
\hline 6 & F2 & 29 & 61 & TOP1 & 11 & 116 & UGCG & 5 \\
\hline 7 & CTNNB1 & 28 & 62 & MET & 11 & 117 & PPARD & 5 \\
\hline 8 & BCL2L1 & 27 & 63 & NR1/2 & 10 & 118 & AURKB & 5 \\
\hline 9 & ACE & 26 & 64 & UTS2R & 10 & 119 & EGLN1 & 4 \\
\hline 10 & MPO & 26 & 65 & PTGER4 & 10 & 120 & CTSV & 4 \\
\hline 11 & HDAC1 & 22 & 66 & FABP1 & 10 & 121 & ODC1 & 4 \\
\hline 12 & HPGDS & 21 & 67 & HMGCR & 10 & 122 & FOLH1 & 4 \\
\hline 13 & P2RY12 & 20 & 68 & AVPR1A & 10 & 123 & PLA2G5 & 4 \\
\hline 14 & CCR5 & 19 & 69 & KCNMA1 & 9 & 124 & ABHD6 & 4 \\
\hline 15 & MGAM & 18 & 70 & PLA2G1B & 9 & 125 & PKN1 & 4 \\
\hline 16 & CXCR1 & 18 & 71 & IDH1 & 9 & 126 & PTPRF & 4 \\
\hline 17 & AGTR1 & 18 & 72 & NTRK1 & 9 & 127 & AKR1B10 & 4 \\
\hline 18 & ABCG2 & 17 & 73 & VDR & 9 & 128 & MAPKAPK2 & 4 \\
\hline 19 & FLT3 & 17 & 74 & MAOA & 9 & 129 & MYLK & 4 \\
\hline 20 & CCR2 & 17 & 75 & CTSA & 9 & 130 & MIF & 3 \\
\hline 21 & CTSB & 17 & 76 & AKR1C3 & 9 & 131 & ESRRB & 3 \\
\hline 22 & PARP1 & 17 & 77 & CASP7 & 9 & 132 & $\mathrm{XDH}$ & 3 \\
\hline 23 & ABCB1 & 16 & 78 & CTSG & 9 & 133 & GABRA2 & 3 \\
\hline 24 & CD38 & 16 & 79 & HPGD & 8 & 134 & ELOVL6 & 3 \\
\hline 25 & ADRA2C & 16 & 80 & ADORA2A & 8 & 135 & KCNK3 & 3 \\
\hline 26 & GRM5 & 16 & 81 & DGAT1 & 8 & 136 & NAAA & 3 \\
\hline 27 & CTSD & 16 & 82 & CES2 & 8 & 137 & SIGMAR1 & 3 \\
\hline
\end{tabular}




\begin{tabular}{|c|c|c|c|c|c|c|c|c|}
\hline No. & Target & Degree & No. & Target & Degree & No. & Target & Degree \\
\hline 28 & CYP2C9 & 16 & 83 & KCNA3 & 8 & 138 & FKBP1A & 3 \\
\hline 29 & MCL1 & 16 & 84 & ADORA2B & 8 & 139 & PKN2 & 3 \\
\hline 30 & LCK & 16 & 85 & SPHK1 & 8 & 140 & CA2 & 3 \\
\hline 31 & GRM2 & 15 & 86 & CAPN1 & 8 & 141 & PYGL & 3 \\
\hline 32 & TLR7 & 15 & 87 & GSR & 8 & 142 & HSD17B2 & 3 \\
\hline 33 & SYK & 15 & 88 & KAT2B & 8 & 143 & $\mathrm{HRH} 2$ & 3 \\
\hline 34 & HNF4A & 15 & 89 & RXRG & 8 & 144 & NAMPT & 3 \\
\hline 35 & PTGS1 & 15 & 90 & $\mathrm{BCHE}$ & 7 & 145 & PIK3CG & 3 \\
\hline 36 & CYP2C19 & 15 & 91 & SLC5A1 & 7 & 146 & YWHAG & 3 \\
\hline 37 & MME & 14 & 92 & GPBAR1 & 7 & 147 & CBR1 & 3 \\
\hline 38 & P2RY1 & 14 & 93 & FDFT1 & 7 & 148 & PLEC & 2 \\
\hline 39 & ADRA1A & 14 & 94 & GBA & 7 & 149 & CA4 & 2 \\
\hline 40 & RARA & 14 & 95 & DPP7 & 7 & 150 & BRD2 & 2 \\
\hline 41 & CALM1 & 14 & 96 & PIM1 & 7 & 151 & HSD11B1 & 2 \\
\hline 42 & SELE & 13 & 97 & PPP1CC & 7 & 152 & ECE1 & 2 \\
\hline 43 & $\mathrm{ACHE}$ & 13 & 98 & CTSS & 7 & 153 & PGGT1B & 2 \\
\hline 44 & S1PR5 & 13 & 99 & EZR & 7 & 154 & KLK1 & 2 \\
\hline 45 & GPR18 & 13 & 100 & FABP3 & 6 & 155 & F13A1 & 2 \\
\hline 46 & NPY2R & 13 & 101 & TRPV4 & 6 & 156 & TAOK3 & 1 \\
\hline 47 & S1PR4 & 13 & 102 & FABP2 & 6 & 157 & CA6 & 1 \\
\hline 48 & DPP4 & 13 & 103 & ALDH2 & 6 & 158 & CA13 & 1 \\
\hline 49 & PTGFR & 13 & 104 & ADH1C & 6 & 159 & CECR2 & 1 \\
\hline 50 & ALOX5 & 13 & 105 & BRAF & 6 & 160 & PPM1B & 1 \\
\hline 51 & ANPEP & 12 & 106 & THRB & 6 & 161 & NAE1 & 1 \\
\hline 52 & CCR8 & 12 & 107 & PTPN22 & 6 & 162 & ERN1 & 1 \\
\hline 53 & $\mathrm{HRH} 4$ & 12 & 108 & ALOX5AP & 6 & 163 & PDE4B & 1 \\
\hline 54 & MAPK9 & 12 & 109 & PTPRS & 5 & 164 & CA1 & 1 \\
\hline 55 & MGLL & 11 & 110 & KCNA5 & 5 & & & \\
\hline
\end{tabular}




\subsection{G0 enrichment analysis}

GO mainly includes three sub-ontologies: biological process (BP), cellular component (CC), and molecular function (MF). We used the org.Hs.eg.db and ClusterProfiler data packages of R software to perform GO enrichment analysis of the common targets obtained. After we retained results for which $P \leq 0.05$, we performed visual analysis on the first 20 items and obtained the histograms shown in Figs. 4-6. In these bar charts, the ordinate represents the items of enrichment, and the abscissa represents the enrichment scores of these terms. Figure 4 shows that of the common targets in BP, the main enrichment items were cellular calcium ion homeostasis, response to steroid hormone, calcium ion homeostasis, unsaturated fatty acid metabolism, and positive regulation of phospholipase activity. Figure 5 illustrates that the main enrichment items of the common targets in CC were membrane raft, membrane microdomain, apical part of cell, membrane region, and presynapse. Figure 6 shows that the main enrichment items of the common targets in MF were serine/threonine-protein kinase activity, G-protein-coupled peptide receptor activity, peptide receptor activity, oxidoreductase activity, acting on the $\mathrm{CH}-\mathrm{OH}$ group of donors, nicotinamide adenine dinucleotide (NAD) or nicotinamide adenine dinucleotide phosphate (NADPH) as acceptor, endopeptidase activity, and protein tyrosine kinase activity. These findings indicated that HSZF might work through multiple biological processes in treating COVID-19.

\subsection{KEGG pathway enrichment analysis}

We performed KEGG pathway enrichment analysis on the common targets obtained using the R software data packages org.Hs.eg.db and ClusterProfiler. Based on retained results for which $P \leq 0.05$, we performed visual analysis to obtain a KEGG enrichment analysis histogram (Fig. 7). The ordinate represents the name of the enrichment pathway, and the abscissa represents the enrichment scores of these terms. The common targets are mainly enriched in related signaling pathways such as neuroactive ligand-receptor interaction, apoptosis, calcium signaling, vascular smooth-muscle contraction, phospholipase D signaling, arachidonic acid metabolism, platelet activation, renin-angiotensin system (RAS), inflammatory-mediator regulation of TRP channels, and renin secretion. This showed that the active constituent targets of HSZF were distributed across different pathways and might work together through these multiple pathways.

\section{Discussion}

Early intervention with TCM can reduce conversion from mild disease to severe and critical disease[20]. The TCM dialectical formula corresponding to each syndrome in the diagnosis and treatment plan has been widely used in clinical practice. However, no clinical research has further explored the pharmacological mechanisms of HSZF in the treatment of COVID-19. In this study, we initially used the TCMSP database to screen the active TCM constituents of HSZF, the SwissTargetPrediction database to predict targets, and Cytoscape software to construct a pharmaceutical active-compound-potential-target network and a PPI network. GO and KEGG pathway enrichment analysis were performed to construct a 
target-signal pathway network diagram so as to discover the main active constituents of the formula, as well as its main targets and regulatory pathways. Our research results are summarized as follows.

Based on analysis of the HSZF active-compound-potential-target network, we found that 10 constituents -pectolinarigenin, genkwanin, 5-Hydroxy-7,4'-dimethoxyflavanon, diosmetin, wogonin, quercetin, kaempferol, pachypodol, luteolin, and herbacetin-were the main active constituents of HSZF. This indicated that flavonoids are important for the mechanism by which HSZF treats this disease. ARDS and acute lung injury (ALI) are the main clinical manifestations of critical COVID-19 illness; their symptoms are dyspnea and hypoxemia. Sepsis caused by Gram-negative bacterial infections often cause ALI, and the main component of endotoxins in the outer membranes of Gram-negative bacteria cell walls is lipopolysaccharide (LPS). LPS is a common endotoxin and inflammation-triggering factor that can activate neutrophils to produce a large number of inflammatory factors. After LPS enters the lung tissue of rats, it can cause edema in the pulmonary interstitium and symptoms such as dyspnea and hypoxemia[21]. Difficulty breathing and MOF, which occur in COVID-19, might be related to endotoxins. Studies have shown that quercetin can protect mice from LPS-induced ALI by inhibiting the nuclear factor $\mathrm{K}$-light-chain-enhancer of activated $\mathrm{B}$ cells (NF-KB) signaling pathway and reducing production of NF-KB and intercellular adhesion molecule 1 (ICAM-1) to achieve anti-inflammatory effects[22]. In addition, quercetin mainly prevents the virus receptor complex from entering cells by antagonizing the calcium ion channel and interrupting its life cycle, thereby causing the virus to die and achieving antiviral effect[23]. Diosmetin can reduce LPS-induced ALI by activating the nuclear factor (erythroid-derived 2)-like 2 (Nrf2) pathway and inhibiting the nucleotide-binding domain-like receptor protein 3 (NLRP3) inflammatory response[24]. Genkwanin has excellent anti-inflammatory effects in vitro. It mainly inhibits the production of pro-inflammatory mediators by regulating the micro-ribonucleic-acid (miR101), microtubuleassociated protein kinase phosphatase-1 (Mkp-1), and mitogen-activated protein kinase (MAPK) pathways in LPS-activated macrophages [25]. Wogonin can inhibit excessive activation of the complement system in vivo and improve ALI induced by influenza A virus[26]. Luteolin has a variety of pharmacological activities, such as anti-inflammatory, anti-allergic, anti-tumor, anti-bacterial, and antiviral. It is mainly used in clinical practice for resolving cough, eliminating phlegm, and combating inflammation[27]. Kaempferol has anti-oxidant activity and can scavenge oxygen free radicals and reduce tissue damage caused by hypoxia[28], as well as inhibit the expression of tumor necrosis factor alpha (TNF-a), IL-6, IL-10, IL-1 $\beta$, vascular cell adhesion protein 1 (VCAM-1), and ICAM- 1 by reducing the activity of the MAKP, NF-KB and other pathways to exert an anti-inflammatory effect[29].

In terms of potential targets, studies have shown that SARS-CoV-2, like SARS-CoV, binds to ACE2 in the body through its expressed $S$ protein, then invades the body and causes illness, leading to severe pneumonia and high mortality[30]. Other potential targets, such as kininogen-1 (KNG1), epidermal growth factor receptor (EGFR), Caspase-3 (CASP3), signal transducer and activator of transcription 3 (STAT3), and myeloperoxidase (MPO), have all been confirmed to be involved in pulmonary inflammation. For example, KNG1 is an inflammatory mediator; triptolide can reduce chronic obstructive pulmonary disease (COPD) by inhibiting the cellular inflammation caused by cigarette smoke through the KNG1 signaling pathway[31]. EGFR transactivation can induce airway epithelial cells to produce mucus and promote 
inflammatory-cytokine secretion[32], and studies have shown that EGFR is associated with airway inflammation in asthmatic rats[33]. CASP3 participates in the activation cascade of caspases and is responsible for initiating apoptosis. In a study involving a mouse model of allergic airway inflammation, quercetin improved chronic histopathological changes by regulating the processes of epithelial-derived cytokines and epithelial-cell apoptosis. In addition to basement membrane thickness in lung tissue, it also had beneficial effects on inflammation[34]. STAT3 transcription factors can activate macrophages and neutrophils and enhance the inflammatory response. Research has shown that inhibition of STAT3 activity can protect against ALI caused by LPS[35]. After hemorrhagic-shock resuscitation in rats, MPO activity is enhanced, decomposing extracellular fibers and matrix, thereby causing inflammatory reactions in lung parenchyma and ALI. Inhaling $2 \%$ hydrogen can reduce such MPO activity as well as infiltration of inflammatory cells into lung tissue, thereby minimizing the degree of lung injury[36]. The abovementioned findings elucidate the potential action mechanisms of the active constituents and targets of HSZF in the treatment of COVID-19, but there are still some highly relevant targets that previous studies did not prove to have antiviral or anti-inflammatory effects. For example, the biological effect of estrogen is currently thought to be mainly regulated by estrogen receptor a $(E R a)$, and the potential target estrogen receptor 1 (ESR1) combined with estrogen can exert antitumor effects[37]. However, there are no reports on ESR1 having anti-inflammatory or lung-protective effects. Our findings provide new predictions for the molecular basis of HSZF in treating COVID-19 and new ideas for further research.

From the results of GO enrichment analysis, it can be seen that the potential targets of HSZF were mainly distributed across cell membranes, neural axons, lysosome cavities, and other areas, with biological functions such as endopeptidase activity; $G$ protein-coupled peptide receptor activity; nuclear receptor activity; oxidoreductase activity; steroid hormone receptor activity; and participation in biological processes such as cellular metal ion homeostasis, calcium ion homeostasis, cellular divalent inorganiccation homeostasis, fatty acid-derivative metabolism, unsaturated fatty acid metabolism, positive regulation of phospholipase activity, ion channel regulation, immune regulation, and chemical-signal transmission. Studies have shown that the hydroxyl group can exert its cytotoxic effect by changing the homeostasis of the cell's calcium ion, causing alveolar macrophages to produce excessive reactive oxygen free radicals, which leads to oxidative lung injury[38]. Inflammation causes disease through fluid transfer across cell membranes and cell layers, which leads to changes in muscle function and pain, and changes in ion channels have been detected in ALI[39]. Neutral endopeptidase is an enzyme that cleaves inflammatory bioactive peptides and plays a protective role in the pathogeneses of ALI and ARDS[40].

From the results of KEGG pathway enrichment analysis, we saw that of the pathways involved in the treatment process, arachidonic acid metabolism was an important inflammatory pathway. It mainly synthesizes inflammatory mediators and mediates the production of multiple inflammatory factors such as monocyte chemotactic protein-1 (MCP-1), TNF, interleukins, and interferon, which are closely related to the development, progression, and resolution of inflammation[41]. Studies have found that SARS-CoV-2 can bind to ACE2 and cause depletion through the receptor-binding domain (RBD) of the S protein, leading to an imbalance in RAS, upregulation of the ACE/angiotensin II (Ang II)/Ang II type 1 receptor 
$\left(A T_{1} R\right)$ pathway, and severe pneumonia[30]. Phospholipase $\mathrm{D}$ is closely related to inflammation; PLD1 and PLD2 cause increased leukocyte chemotaxis and inflammation[42]. Platelet activation is also one of the main pathways involved in the treatment process. Platelets play a central role in maintaining coagulation homeostasis, as they are also involved in immune responses and host defenses[43]; and that chemokine (C-X3-C motif) ligand 7 ( $C X C L 7)$, which induces chemotaxis and activation of neutrophils, and chemokine (C-X3-C motif) ligand 4 (CXCL4), which induces neither, promote ALI via complementary effects and activation of vascular permeability[44]. TRP channel proteins regulate various physiological activities of cells, such as autophagy and apoptosis, by regulating $\mathrm{Ca}^{2+}[45]$. It is speculated that HSZF treats COVID-19 by inhibiting inflammatory response and regulating both immune function and apoptosis.

In summary, we used network pharmacology techniques and methods to retrieve active TCM constituents and potential targets of HSZF, screened active constituents of drugs and common targets between active constituents and COVID-19, and performed GO and KEGG pathway enrichment analysis on the targets. We speculated that the active constituents of HSZF in COVID-19 treatment might be genkwanin, diosmetin, wogonin, quercetin, kaempferol, and luteolin; and the potential targets might be ACE2, KNG1, EGFR, CASP3, MPO, and STAT3. The abovementioned active components regulate signaling pathways such as arachidonic acid metabolism, platelet activation, phospholipase D, RAS, and inflammatorymediator regulation of TRP channels to inhibit inflammatory response, regulate immune function, regulate cell apoptosis, and reduce lung injury, so as to achieve the purpose of treating COVID-19.

\section{Declarations}

\section{Ethics approval and consent to participate}

Not applicable. This manuscript does not report on or involve the use of any animal or human data or tissue.

\section{Consent for publication}

Not applicable. This manuscript does not contain data from any individual person.

\section{Availability of data and materials}

All data are available in the manuscript and are shown in tables, figures.

\section{Competing interests}

The authors declare that they have no conflicts of interest.

\section{Funding}


This work is supported by National Natural Science Foundation of China (No.81973814), the Liu Xiaohong Famous Traditional Chinese Medicine Inheritance Studio from theTraditional Chinese Medicine Bureau of Guangdong Province (No.201805), the Construction Project of the National Clinical Medical Research Center (respiratory department, No.2110200309), the Construction Project of High Level Hospital on Modified Weijing Granules (No.211010010603), and the Student Learning Team Incubation Project of Innovation Academy from The First Affiliated Hospital of Guangzhou University of Chinese Medicine (No.2018XXTD003), and the Guangdong Provincial Science and Technology Plan Project (South China Traditional Chinese Medicine Collaborative Innovation Center, No.2014b0902002), and Department of Science and Technology of Guangdong Province (No. 2020B111110001), COVID-19 Epidemic Prevention and Control Special Research Fund of the Department of Education of Guangdong Province (No.2020KZDZX1054) and Student Learning Team Incubation Project of the First Affiliated Hospital of Guangzhou University of Traditional Chinese Medicine (No.2018XXTD003).

\section{Authors' Contributions}

Wen-jiang Zheng and Yong-shi Ni conceived and designed the research; Wen-jiang Zheng collected and analyzed the data; Ji-qiang Li, Xiao-hong Liu, and Yong Jiang provided useful suggestions on the methodology; Yong-shi Ni, Wen-jiang Zheng and Qian Yan wrote the paper. All authors read and approved the final version of the manuscript. Yong-shi Ni and Wen-jiang Zheng contributed equally to this work, and should be regarded as co-first authors.

\section{Acknowledgments}

We thank LetPub for its linguistic assistance during the preparation of this manuscript.

\section{References}

1. Chan JF, Yuan S, Kok KH, To KK, Chu H, Yang J, et al. A familial cluster of pneumonia associated with the 2019 novel coronavirus indicating person-to-person transmission: a study of a family cluster. Lancet. 2020;395(10223):514-23. doi:10.1016/S0140-6736(20)30154-9.

2. Phan LT, Nguyen TV, Luong QC, Nguyen TV, Nguyen HT, Le HQ, et al. Importation and Human-toHuman Transmission of a Novel Coronavirus in Vietnam. N Engl J Med. 2020;382(9):872-4. doi:10.1056/NEJMc2001272.

3. Wang D, Hu B, Hu C, Zhu F, Liu X, Zhang J, et al. Clinical Characteristics of 138 Hospitalized Patients With 2019 Novel Coronavirus-Infected Pneumonia in Wuhan, China. JAMA. 2020. doi:10.1001/jama.2020.1585.

4. Zhang MM, Liu XM, He L. Effect of integrated traditional Chinese and Western medicine on SARS: a review of clinical evidence. World J Gastroenterol. 2004;10(23):3500-5. doi:10.3748/wjg.v10.i23.3500.

5. China NHCotPsRo. Coronavirus Disease 2019 (COVID-19) diagnosis and treatment plan (in Chinese, 7th version). 2020. 
http://www.nhc.gov.cn/yzygj/s7653p/202003/46c9294a7dfe4cef80dc7f5912eb1989.shtml.

6. Hopkins AL. Network pharmacology: the next paradigm in drug discovery. Nat Chem Biol. 2008;4(11):682-90. doi:10.1038/nchembio.118.

7. Zhao J, Lv C, Wu Q, Zeng H, Guo X, Yang J, et al. Computational systems pharmacology reveals an antiplatelet and neuroprotective mechanism of Deng-Zhan-Xi-Xin injection in the treatment of ischemic stroke. Pharmacol Res. 2019;147:104365. doi:10.1016/j.phrs.2019.104365.

8. Ru J, Li P, Wang J, Zhou W, Li B, Huang C, et al. TCMSP: a database of systems pharmacology for drug discovery from herbal medicines. J Cheminform. 2014;6:13. doi:10.1186/1758-2946-6-13.

9. Alqahtani S. In silico ADME-Tox modeling: progress and prospects. Expert Opin Drug Metab Toxicol. 2017;13(11):1147-58. doi:10.1080/17425255.2017.1389897.

10. Liu J, Pei T, Mu J, Zheng C, Chen X, Huang C, et al. Systems Pharmacology Uncovers the Multiple Mechanisms of Xijiao Dihuang Decoction for the Treatment of Viral Hemorrhagic Fever. Evid Based Complement Alternat Med. 2016;2016:9025036. doi:10.1155/2016/9025036.

11. Wang N, Zheng Y, Gu J, Cai Y, Wang S, Zhang F, et al. Network-pharmacology-based validation of TAMS/CXCL-1 as key mediator of XIAOPI formula preventing breast cancer development and metastasis. Sci Rep. 2017;7(1):14513. doi:10.1038/s41598-017-15030-3.

12. Daina A, Michielin O, Zoete V. SwissTargetPrediction: updated data and new features for efficient prediction of protein targets of small molecules. Nucleic Acids Res. 2019;47(W1):W357-W64.

13. $10.1101 / 2020.02 .05 .20020545$

Jun Wang SZ, Ming Liu Z, Zhao Y, Xu P, Wang M, Lin Y, Xu B, Huang X, Zuo Z, Chen F, Bai J, Cui AM, Lew J, Zhao Y, Zhang H, Luo Y Zhang. ACE2 expression by colonic epithelial cells is associated with viral infection, immunity and energy metabolism. medRxiv. 2020; doi:

https://doi.org/10.1101/2020.02.05.20020545.

14. Su G, Morris JH, Demchak B, Bader GD. Biological network exploration with Cytoscape 3. Curr Protoc Bioinformatics. 2014;47:8.13.1-24..

15. Szklarczyk D, Morris JH, Cook H, Kuhn M, Wyder S, Simonovic M, et al. The STRING database in 2017: quality-controlled protein-protein association networks, made broadly accessible. Nucleic Acids Res. 2017;45(D1):D362-D8. doi:10.1093/nar/gkw937.

16. Chin $\mathrm{CH}$, Chen $\mathrm{SH}, \mathrm{Wu} \mathrm{HH}, \mathrm{Ho} \mathrm{CW}$, Ko MT, Lin CY. cytoHubba: identifying hub objects and subnetworks from complex interactome. BMC Syst Biol. 2014;8(Suppl 4):11. doi:10.1186/1752-0509-8S4-S11.

17. Yu G, Wang LG, Han Y, He QY. clusterProfiler: an R package for comparing biological themes among gene clusters. OMICS. 2012;16(5):284-7.

18. Ashburner M, Ball CA, Blake JA, Botstein D, Butler H, Cherry JM, et al. Gene ontology: tool for the unification of biology. The Gene Ontology Consortium. Nat Genet. 2000;25(1):25-9. doi:10.1038/75556.

19. Kanehisa M, Goto S, Kawashima S, Okuno Y, Hattori M. The KEGG resource for deciphering the genome. Nucleic Acids Res. 2004;32(Database issue):D277-80. doi:10.1093/nar/gkh063. 
20. Chan KW, Wong VT, Tang SCW. COVID-19: An Update on the Epidemiological, Clinical, Preventive and Therapeutic Evidence and Guidelines of Integrative Chinese-Western Medicine for the Management of 2019 Novel Coronavirus Disease. Am J Chin Med. 2020:1-26; doi:

10.1142/S0192415X20500378.

21. Shaaban AA, El-Kashef DH, Hamed MF, El-Agamy DS. Protective effect of pristimerin against LPSinduced acute lung injury in mice. Int Immunopharmacol. 2018;59:31-9. doi:10.1016/j.intimp.2018.03.033.

22. Takashima K, Matsushima M, Hashimoto K, Nose H, Sato M, Hashimoto N, et al. Protective effects of intratracheally administered quercetin on lipopolysaccharide-induced acute lung injury. Respir Res. 2014;15:150. doi:10.1186/s12931-014-0150-x.

23. Wu SN, Chiang HT, Shen AY, Lo YK. Differential effects of quercetin, a natural polyphenolic flavonoid, on L-type calcium current in pituitary tumor (GH3) cells and neuronal NG108-15 cells. J Cell Physiol. 2003;195(2):298-308. doi:10.1002/jcp.10244.

24. Liu Q, Ci X, Wen Z, Peng L. Diosmetin Alleviates Lipopolysaccharide-Induced Acute Lung Injury through Activating the Nrf2 Pathway and Inhibiting the NLRP3 Inflammasome. Biomol Ther (Seoul). 2018;26(2):157-66. doi:10.4062/biomolther.2016.234.

25. Gao Y, Liu F, Fang L, Cai R, Zong C, Qi Y. Genkwanin inhibits proinflammatory mediators mainly through the regulation of miR-101/MKP-1/MAPK pathway in LPS-activated macrophages. PLoS One. 2014;9(5):e96741. doi:10.1371/journal.pone.0096741.

26. Zhi H, Jin X, Zhu H, Li H, Zhang Y, Lu Y, et al. Exploring the effective materials of flavonoids-enriched extract from Scutellaria baicalensis roots based on the metabolic activation in influenza $A$ virus induced acute lung injury. J Pharm Biomed Anal. 2020;177:112876. doi:10.1016/j.jpba.2019.112876.

27. Birt DF, Hendrich S, Wang W. Dietary agents in cancer prevention: flavonoids and isoflavonoids. Pharmacol Ther. 2001;90(2-3):157-77. doi:10.1016/s0163-7258(01)00137-1.

28. Imran M, Rauf A, Shah ZA, Saeed F, Imran A, Arshad MU, et al. Chemo-preventive and therapeutic effect of the dietary flavonoid kaempferol: A comprehensive review. Phytother Res. 2019;33(2):26375. doi:10.1002/ptr.6227.

29. Dong X, Fu J, Yin X, Cao S, Li X, Lin L, et al. Emodin: A Review of its Pharmacology, Toxicity and Pharmacokinetics. Phytother Res. 2016;30(8):1207-18. doi:10.1002/ptr.5631.

30. Sun ML, Yang JM, Sun YP, Su GH. [Inhibitors of RAS Might Be a Good Choice for the Therapy of COVID-19 Pneumonia]. Zhonghua Jie He He Hu Xi Za Zhi. 2020;43(3):219-22. doi:10.3760/cma.j.issn.1001-0939.2020.03.016.

31. Shi K, Chen X, Xie B, Yang SS, Liu D, Dai G, et al. Celastrol Alleviates Chronic Obstructive Pulmonary Disease by Inhibiting Cellular Inflammation Induced by Cigarette Smoke via the Ednrb/Kng1 Signaling Pathway. Front Pharmacol. 2018;9:1276. doi:10.3389/fphar.2018.01276.

32. Shimizu S, Takezawa-Yasuoka K, Ogawa T, Tojima I, Kouzaki H, Shimizu T. The epidermal growth factor receptor inhibitor AG1478 inhibits eosinophilic inflammation in upper airways. Clin Immunol. 2018;188:1-6. doi:10.1016/j.clim.2017.11.010. 
33. Long HC, Wang ZL, Xiao BR, Li XH, Li W, Zhou YT. [Effects of epidermal growth factor receptor on airway inflammation in a rat asthmatic model]. Zhonghua Jie He He Hu Xi Za Zhi. 2009;32(7):51721.

34. Caglayan Sozmen S, Karaman M, Cilaker Micili S, Isik S, Bagriyanik A, Arikan Ayyildiz Z, et al. Effects of Quercetin Treatment on Epithelium-derived Cytokines and Epithelial Cell Apoptosis in Allergic Airway Inflammation Mice Model. Iran J Allergy Asthma Immunol. 2016;15(6):487-97.

35. Zhao J, Yu H, Liu Y, Gibson SA, Yan Z, Xu X, et al. Protective effect of suppressing STAT3 activity in LPS-induced acute lung injury. Am J Physiol Lung Cell Mol Physiol. 2016;311(5):L868-L80. doi:10.1152/ajplung.00281.2016.

36. Moon DH, Kang DY, Haam SJ, Yumoto T, Tsukahara K, Yamada T, et al. Hydrogen gas inhalation ameliorates lung injury after hemorrhagic shock and resuscitation. J Thorac Dis. 2019;11(4):151927. doi:10.21037/jtd.2019.03.23.

37. Lei JT, Gou X, Seker S, Ellis MJ. ESR1 alterations and metastasis in estrogen receptor positive breast cancer. J Cancer Metastasis Treat. 2019;5; doi:10.20517/2394-4722.2019.12.

38. Rojanasakul Y, Wang L, Hoffman AH, Shi X, Dalal NS, Banks DE, et al. Mechanisms of hydroxyl free radical-induced cellular injury and calcium overloading in alveolar macrophages. Am J Respir Cell Mol Biol. 1993;8(4):377-83. doi:10.1165/ajrcmb/8.4.377.

39. Eisenhut M, Wallace H. Ion channels in inflammation. Pflugers Arch. 2011;461(4):401-21. doi:10.1007/s00424-010-0917-y.

40. Hashimoto S, Amaya F, Oh-Hashi K, Kiuchi K, Hashimoto S. Expression of neutral endopeptidase activity during clinical and experimental acute lung injury. Respir Res. 2010;11:164. doi:10.1186/1465-9921-11-164.

41. Sala A, Proschak E, Steinhilber D, Rovati GE. Two-pronged approach to anti-inflammatory therapy through the modulation of the arachidonic acid cascade. Biochem Pharmacol. 2018;158:161-73. doi:10.1016/j.bcp.2018.10.007.

42. Gomez-Cambronero J. New concepts in phospholipase D signaling in inflammation and cancer. ScientificWorldJournal. 2010;10:1356-69. doi:10.1100/tsw.2010.116.

43. de Stoppelaar SF, van 't Veer C, van der Poll T. The role of platelets in sepsis. Thromb Haemost. 2014;112(4):666-77. doi:10.1160/TH14-02-0126.

44. Bdeir K, Gollomp K, Stasiak M, Mei J, Papiewska-Pajak I, Zhao G, et al. Platelet-Specific Chemokines Contribute to the Pathogenesis of Acute Lung Injury. Am J Respir Cell Mol Biol. 2017;56(2):261-70. doi:10.1165/rcmb.2015-02450C.

45. Fliniaux I, Germain E, Farfariello V, Prevarskaya N. TRPs and $\mathrm{Ca}(2+)$ in cell death and survival. Cell Calcium. 2018;69:4-18. doi:10.1016/j.ceca.2017.07.002.

\section{Figures}




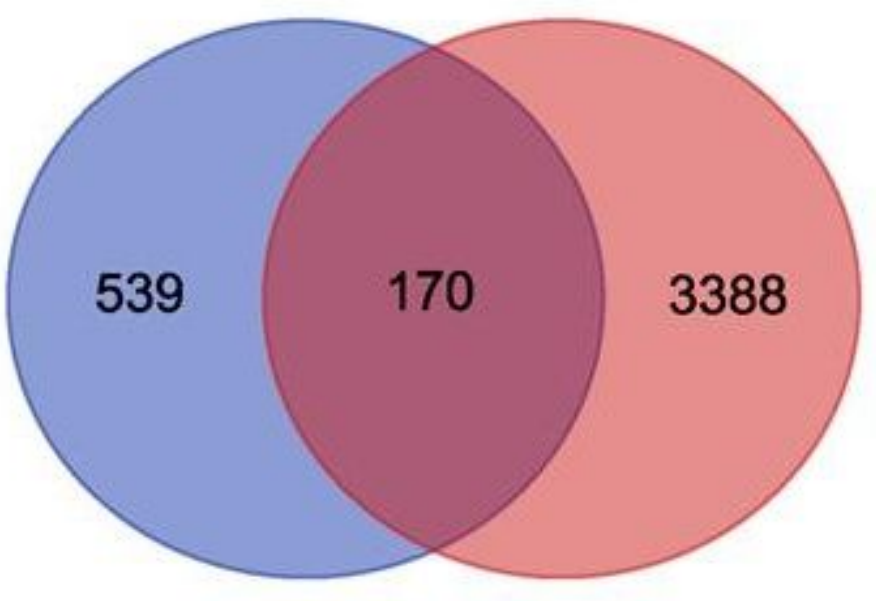

Figure 1

A total of 709 potential targets of Hanshi Zufei syndrome formula (HSZF) and 3558 potential targets for COVID-19, with 170 overlapped targets in total.

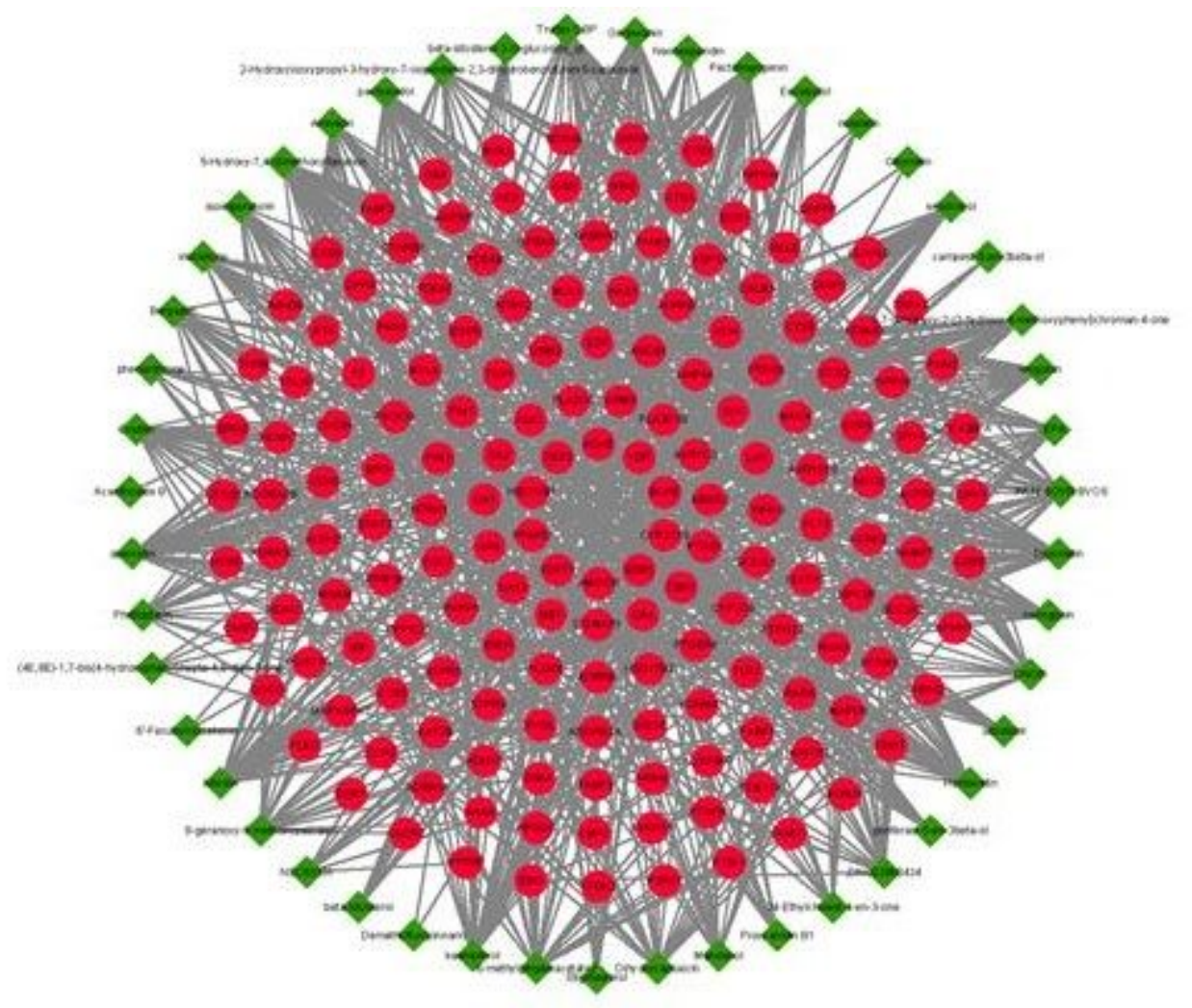

Figure 2

Compound-target network diagram (created in Cytoscape software version 3.7.0). Green represents compounds; red represents targets. 


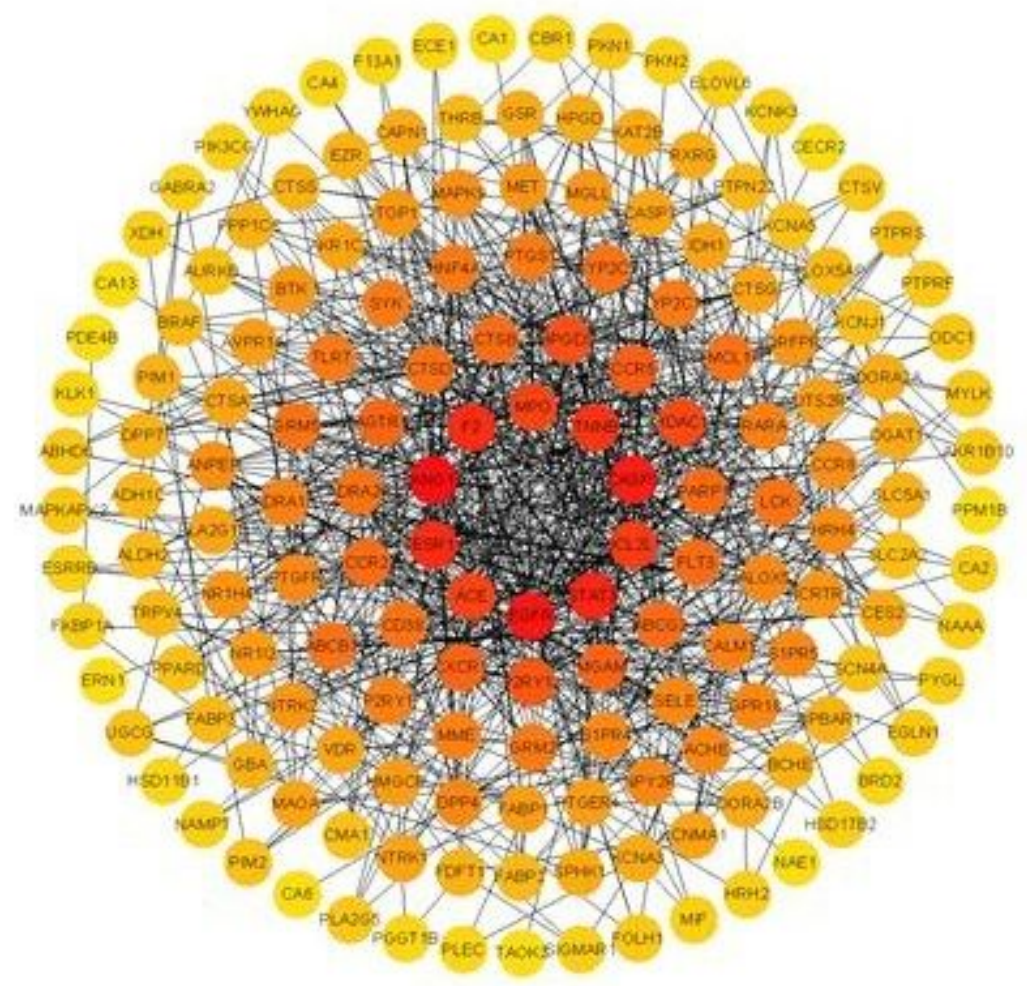

Figure 3

Protein-protein interaction (PPI) network diagram. 
fatty acid derivative metabolic process cellular metal ion homeostasis regulation of cytosolic calcium ion concentration regulation of tube diameter regulation of blood vessel diameter icosanoid biosynthetic process divalent inorganic cation homeostasis cellular calcium ion homeostasis response to steroid hormone regulation of blood vessel size regulation of tube size vascular process in circulatory system calcium ion homeostasis

cellular divalent inorganic cation homeostasis unsaturated fatty acid metabolic process positive regulation of phospholipase activity fatty acid derivative biosynthetic process modulation of chemical synaptic transmission regulation of trans-synaptic signaling
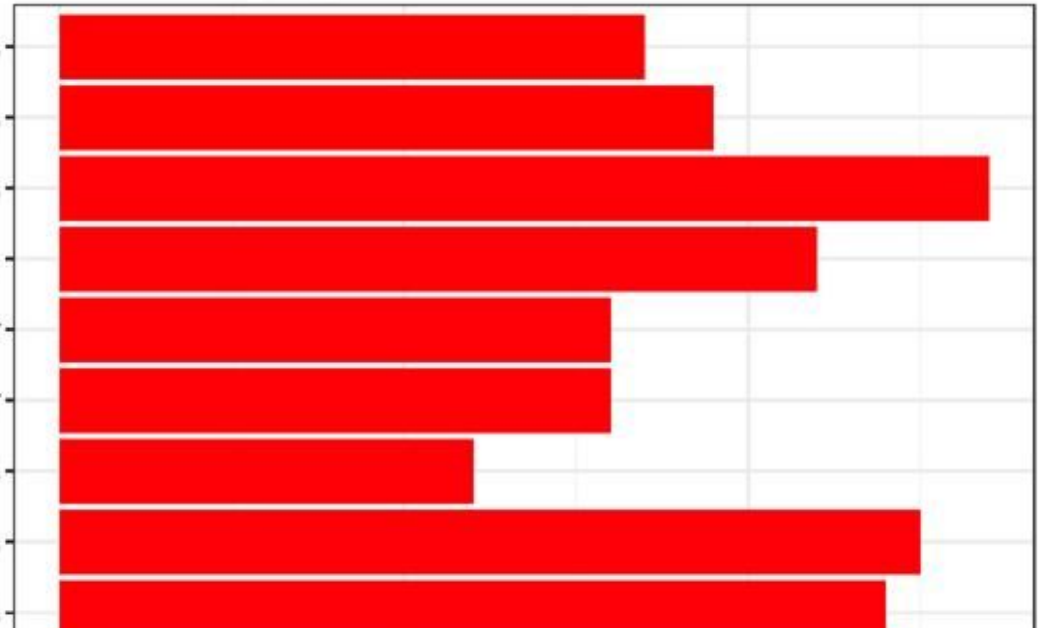

\section{Figure 4}

Biological process (BP) bar chart of the main targets of HSZF. 


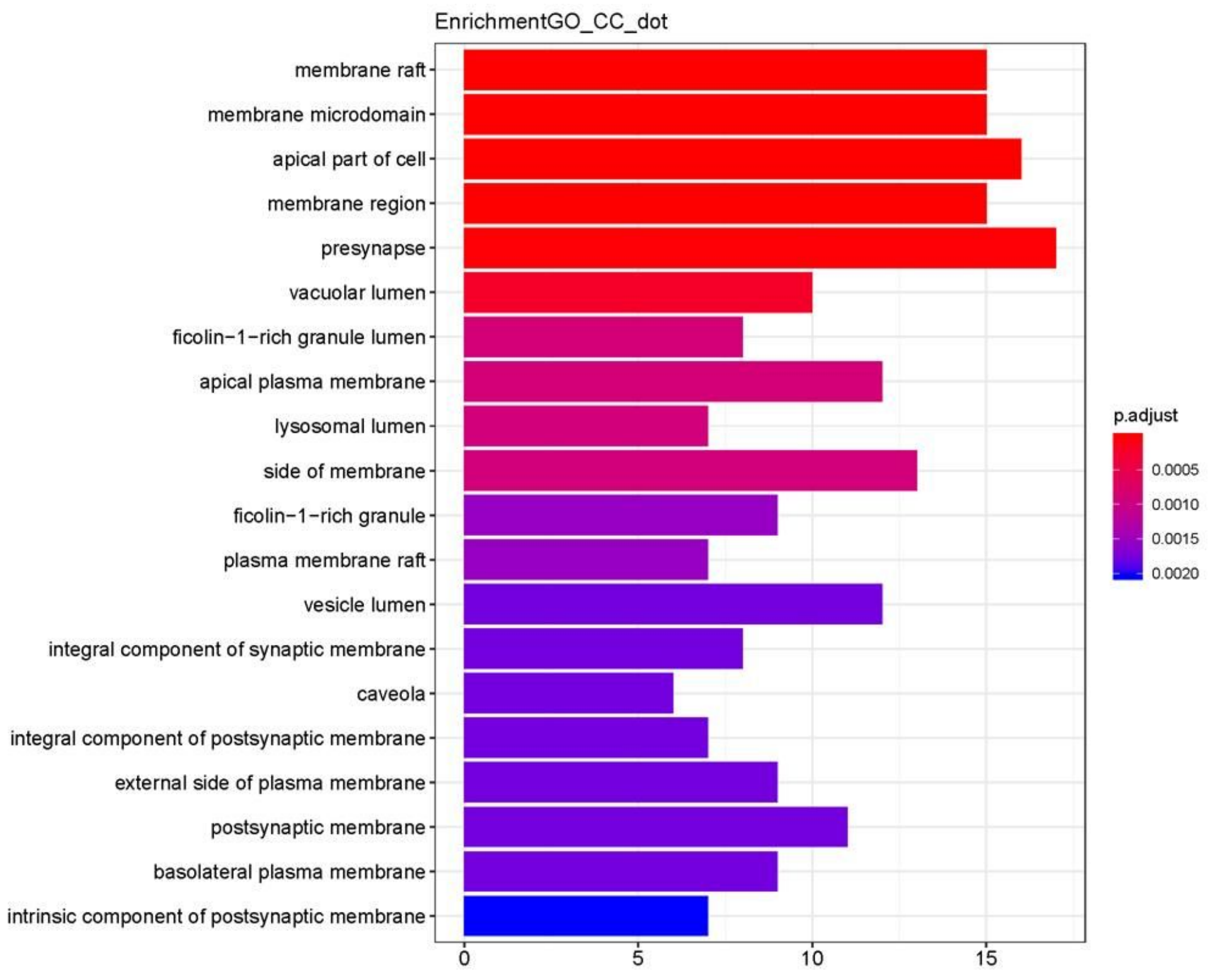

\section{Figure 5}

Cellular component (CC) bar chart of the main targets of HSZF. 


monocarboxylic acid binding

transcription factor activity, direct ligand regulated sequence-specific DNA binding steroid hormone receptor activity -

bile acid binding carboxylic acid binding organic acid binding carbonate dehydratase activity fatty acid binding G protein-coupled adenosine receptor activity protein serine/threonine kinase activity bile acid receptor activity G protein-coupled peptide receptor activity peptide receptor activity oxidoreductase activity, acting on the $\mathrm{CH}-\mathrm{OH}$ group of donors, NAD or NADP as acceptorprotein tyrosine kinase activity oxidoreductase activity, acting on $\mathrm{CH}-\mathrm{OH}$ group of donorscarboxylic ester hydrolase activity long-chain fatty acid binding endopeptidase activity -
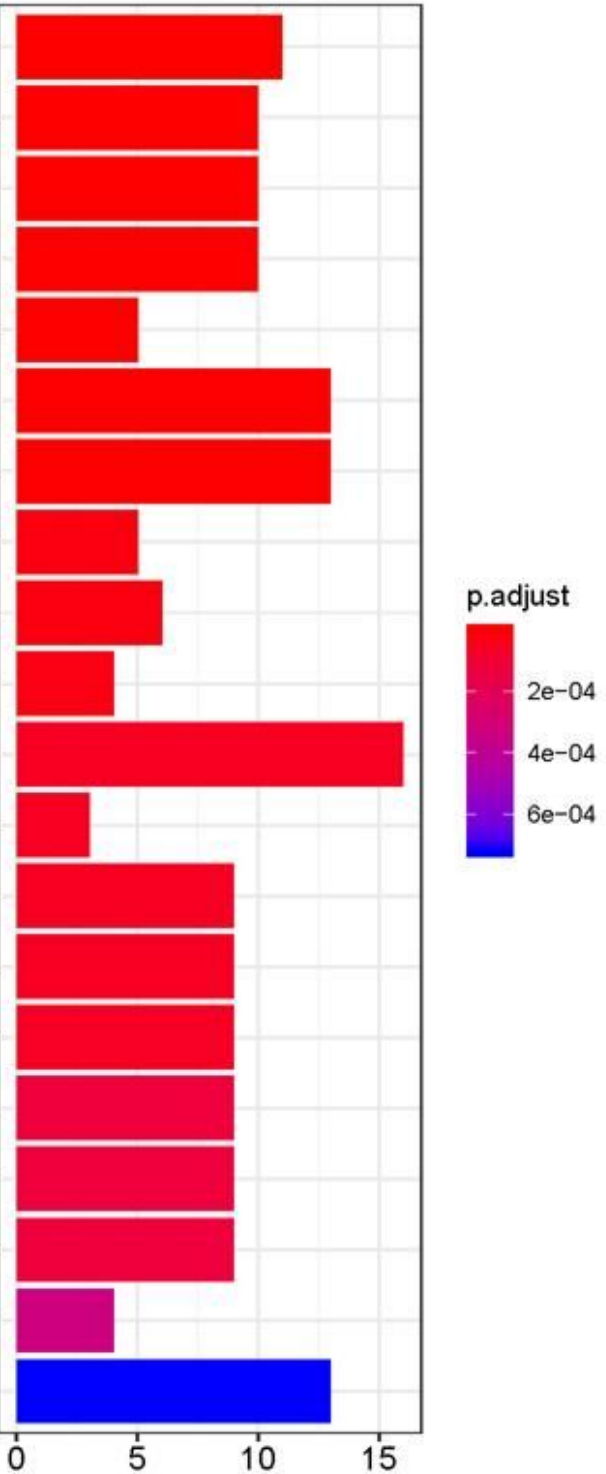

\section{Figure 6}

Molecular function (MF) bar chart of the main targets of HSZF. 


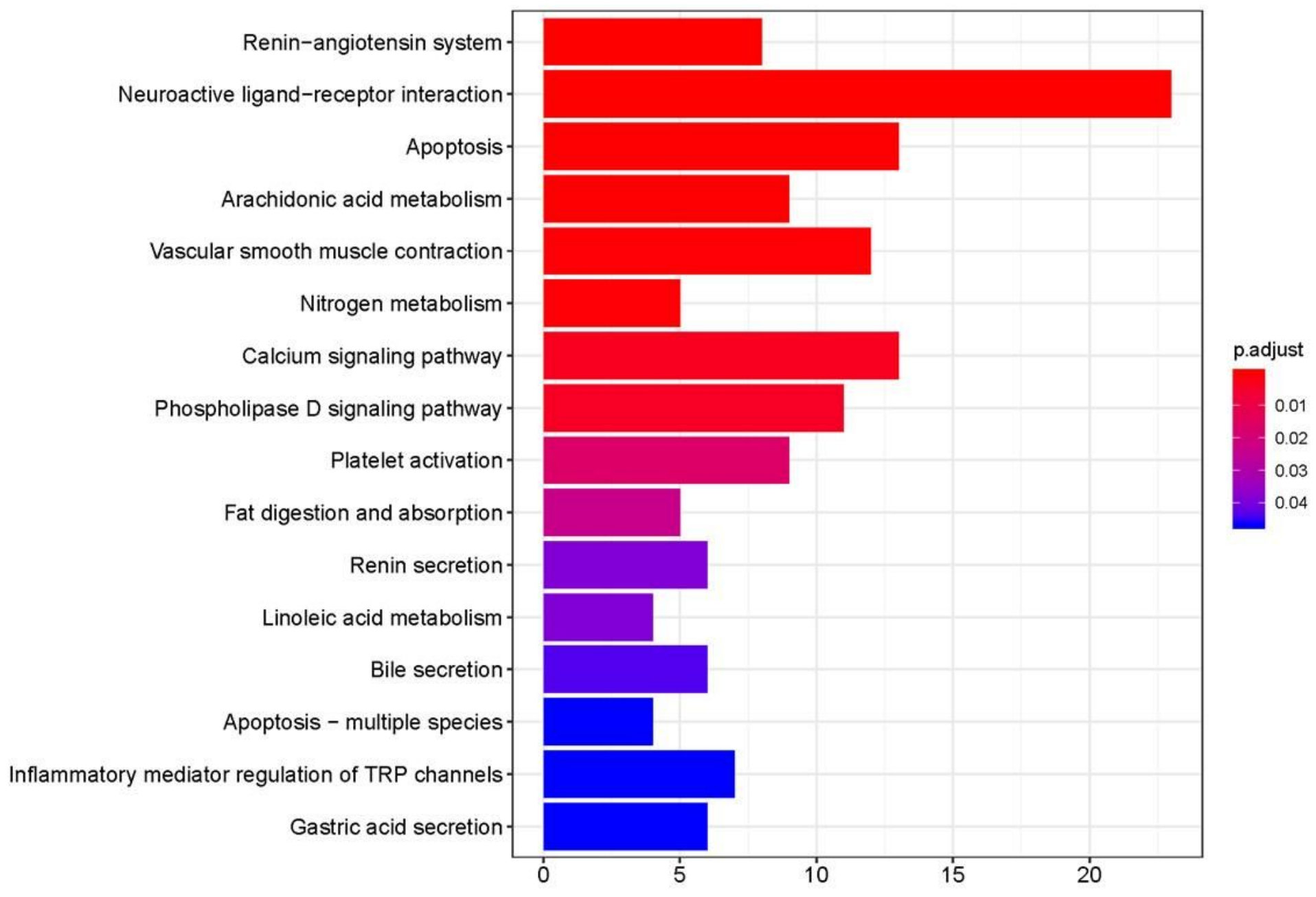

\section{Figure 7}

Histogram of KEGG pathway enrichment analysis of the main targets of HSZF.

\section{Supplementary Files}

This is a list of supplementary files associated with this preprint. Click to download.

- KEGG.csv

- GO.csv 